\title{
Estruturalismo na Filosofia da Matemática e a teoria das categorias: Um debate entre Steve Awodey e Geofrey Hellman
}

\author{
Structuralism in Philosophy of Mathematics and the category theory: \\ A debate between Steve Awodey and Geofrey Hellman
}

do $10.21680 / 1983-2109.2021 v 28 n 57$ ID24120

\author{
Igor Souza Saraiva \\ Universidade Federal de Goiás (UFG) \\ 0000-0002-4055-8653 \\ igorss-uru@hotmail.com
}

\begin{abstract}
Resumo: Há alguns anos ocorre um interessante debate concernindo a capacidade da teoria das categorias em fornecer um esquema conceitual autônomo para uma abordagem estruturalista da filosofia da matemática. O ponto de partida da discussão pode ser remetido ao artigo de Steve Awodey (1996), no qual sugere-se que a teoria das categorias é capaz de fornecer uma noção precisa e flexível de 'estrutura' para os propósitos de uma filosofia estruturalista. Geofrey Hellman (2003), principal proponente de uma forma alternativa de estruturalismo matemático (estruturalismo modal), examinou a possibilidade de uma tal sugestão e chegou a uma conclusão parcialmente negativa. Duas principais réplicas, resultantes de perspectivas filosóficas diametralmente opostas, foram sugeridas por Steve Awodey (2004) e Colin Maclarty (2004). No presente artigo, expomos esse debate, bem como examinamos e defendemos a posição de Awodey com nossos próprios argumentos.
\end{abstract}

Palavras-chave: Estruturalismo, Teoria das Categorias, Fundamentos da Matemática.

\begin{abstract}
For some years now there has been an interesting debate concerning the ability of category theory to provide an autonomous conceptual framework for a structuralist approach to the philosophy of mathematics. The starting point of the discussion can be referred to Steve Awodey's (1996) paper, in which he suggests that category theory is capable of providing an accurate and flexible notion of 'structure' for the purposes of a structuralist philosophy. Geofrey Hellman (2003), the main proponent of an alternative form of mathematical structuralism (modal structuralism), examined the possibility of such a suggestion and came to a partially negative conclusion. Two main replicas, resulting from diametrically opposed philosophical perspectives, were suggested by Steve Awodey (2004) and Colin Maclarty (2004). We will expose this debate, as well as examine and defend Awodey's position with our own arguments.
\end{abstract}

Keywords: Structuralism, Category Theory, Foundations of Mathematics. 


\section{INTRODUÇÃO}

'Estruturalismo' designa, dentro da ontologia e epistemologia da matemática, um leque de diferentes abordagens filosóficas que possuem em comum o lema segundo o qual matemática é sobre estruturas abstratas instanciadas em diferentes sistemas estruturados. Todas essas diferentes abordagens compartilham também uma preocupação com alguns problemas filosóficos. Dentre esses, é particularmente influente a indeterminação da referência dos termos numéricos usualmente atribuída a Benacerraf (1965) e Parsons (1964).

Como é bem conhecido, podemos empregar teorias de conjuntos para construir modelos para a aritmética. Qualquer modelo dos números naturais deve consistir em uma sequência dotada de um elemento inicial e uma regra de progressão que satisfaça todos os axiomas de Peano. Existem pelo menos duas maneiras distintas já bem consagradas de se interpretar a teoria dos números naturais dentro das teorias de conjuntos. Ambas as interpretações resultam em um conjunto dos números naturais distinto (ordinais de Zermelo e ordinais de Von Neumman), porém, ambos são isomorfos entre si. De forma mais geral, qualquer modelo que satisfaça os axiomas de Peano em segunda ordem é isomorfo a qualquer outro modelo ${ }^{1}$. Na medida em que para que uma opção seja aceitável é necessário e suficiente que ela satisfaça os axiomas de Peano, as duas opções que mencionamos acima são igualmente aceitáveis. Se defendermos que números são conjuntos, ou que a totalidade dos números naturais é um conjunto, então devemos estar lidando com conjuntos determinados, já que todo conjunto é um conjunto determinado. Então, temos de enfrentar o dilema por qual das muitas opções igualmente aceitáveis é a única opção correta.

Apesar de termos escolhido, como fez Benacerraf, o exemplo particular dos ordinais de Zermelo e dos ordinais de Von Neumman, qualquer construção dos números naturais como classes ou como classes de classes sofre exatamente dos mesmos males. O objetivo do dilema tal como apresentado por Benacerraf não é vetar que números naturais sejam tratados como certas construções dentro de certas teorias, ou mesmo que se professe que uma certa série de conjuntos possa funcionar como uma série de números naturais dentro de algum sistema. Tanto Benacerraf quanto Parsons desenvolveram seus argumentos como uma objeção à teoria dos números de Frege e alguns de seus pressupostos filosóficos. Apontar

\footnotetext{
${ }^{1}$ A restrição “em segunda ordem” tem de ser adicionada pois, como é conhecido pelo teorema de LowenheimSkolem, nenhuma teoria em primeira ordem com modelos infinitos pode ser categórica, ou seja, possuir um único modelo a menos de um isomorfismo.
} 
para o fato de que diversas reduções são não só possíveis por princípio como também igualmente legítimas é uma estratégia que mina a pretensão filosófica de "revelar" o que os números realmente são por meio de alguma redução em termos de conjuntos.

Benacerraf conclui, drasticamente, que números não só não são conjuntos, como também não são propriamente "objetos" de qualquer tipo. Uma vez que são possíveis uma infinidade de reduções, a aritmética seria uma ciência universal que trata igualmente de qualquer sistema de objetos arranjados como uma progressão recursiva, e não uma teoria que descreve uma série particular de objetos. Essa conclusão é uma particularização para o campo da aritmética do lema estruturalista geral que mencionamos no primeiro parágrafo.

Também é bastante comum argumentar em favor de que uma concepção estruturalista é adequada pois ela faz justiça à atividade dos matemáticos praticantes. Quando lidamos com álgebra, por exemplo, é frequente que duas construções feitas, por assim dizer, por "caminhos diferentes" se revelem como isomorfas entre si. Dado um espaço $S$ e um ponto so $\in S$ pode-se determinar um grupo chamado de 'o grupo fundamental de $S$ ' e denotado por $\pi<S$, so $>$. Os elementos desse grupo são todas as transformações representando trajetórias ${ }^{2}$ continuas de so para so. Descrevendo o grupo de forma intuitiva: o elemento neutro do grupo é a trajetória trivial so $\rightarrow$ so que representa a operação de "ficar parado"; a inversa de uma certa trajetória é obviamente "fazer o caminho de volta"; e a multiplicação do grupo é a concatenação de duas trajetórias nas quais o ponto final de uma coincide com o ponto inicial de outra (pela definição de grupo todas as trajetórias acontecem de ser concatenáveis). Se aplicarmos essa definição geral ao círculo $S_{\circ}$ junto com algum ponto o qualquer do círculo, temos que o grupo $\pi<\mathrm{S}_{\circ}$, o> é formado pelos loops que percorrem o círculo $\mathrm{n}$ vezes começando e terminando em $\mathrm{o}$. A concatenação de um loop que percorre o ć́rculo n vezes e um outro que percorre $m$ vezes é um loopque percorre o círculo $n+m$ vezes. Considere que a operação de realizar um único loop ao longo do círculo pode ser feita tanto em sentido horário quanto em sentido anti-horário. Represente uma dessas ações como resultado de se somar 1 e outra como subtrair 1. Temos que o grupo fundamental do círculo é isomorfo ao grupo aditivo formado pelos números inteiros e pela operação de soma denotado por $<\mathbb{Z},+>$. Tanto quanto diga respeito a teoria dos grupos, $\pi<S_{\circ}, 0>$ e $<\mathbb{Z}$, $+>$ são "idênticos". Ambos são o grupo abeliano livremente gerado a partir de um conjunto unitário. $\mathrm{O}$ algebrista praticante não

\footnotetext{
${ }^{2}$ Essas trajetórias são consideradas a menos de uma homotopia, mas isso não parece relevante pra nosso argumento agora.
} 
precisa se incomodar em distinguir duas cópias isomorfas de uma mesma estrutura algébrica.

Temos aqui uma situação que não é completamente diferente do problema das múltiplas reduções apresentado por Parsons e Benacerraf. Números inteiros podem ser introduzidos a partir dos números naturais já construídos, como classes de equivalência de pares ordenados de naturais, definidas pela relação de equivalência $R(<a, b>,<c, d>$ ) verdadeira de dois pares de naturais $<a, b>$ e $<c, d>$ se, e somente se, $a+d=b+c$. Desse modo, um número inteiro é o resultado de se subtrair do primeiro elemento de um par o segundo elemento do mesmo par e a equivalência é introduzida para garantir que, por exemplo, $<1,4>$ e $<2,5>$ denotem o mesmo número inteiro. Assim, podemos falar que existe uma construção dos números inteiros a partir dos ordinais de Zermelo e outra construção a partir dos ordinais de Von Neumman. Na construção dos inteiros a partir dos ordinais de Zermelo, o par ordenado $<\{\varnothing\},\{\{\varnothing\}\}>$ pertence ao número inteiro -1, na construção a partir dos ordinais de Von Neumman não. Vamos usar os índices ' $z$ ' e 'n' para distinguir entre esses dois conjuntos de números inteiros: $\mathbb{Z}_{\mathrm{z}} \mathrm{e} \mathbb{Z}_{\mathrm{n}}$; e devemos fazer o mesmo para distinguir entres as duas operações de soma sobre inteiros $t_{z} \mathrm{e}+_{n}$. Temos agora dois grupos abelianos $\left\langle\mathbb{Z}_{\mathrm{z}},+_{\mathrm{z}}>\right.$ e $\left\langle\mathbb{Z}_{\mathrm{n}},+_{\mathrm{n}}\right\rangle$. Qual dos dois grupos é o verdadeiro grupo dos números inteiros sob a operação de soma? Ou talvez deveríamos construir a aritmética inteira a partir da geometria e dizer que o verdadeiro grupo $<\mathbb{Z}$, +> é o grupo fundamental do círculo?

É trivial dizer que teorias algébricas lidam apenas com a estrutura universal compartilhada por diversos sistemas, já que, ao contrário da aritmética, tais teorias não são formuladas com qualquer símbolo que possa à primeira vista ser tratado como um nome particular, mas somente com termos gerais. Cada teoria algébrica lida universalmente com uma classe geral de estruturas formadas por um domínio e uma lista de operações, agrupadas sobre a mesma teoria por satisfazerem uma mesma lista de equações. Mas a aritmética pode vir a ser descrita como se fosse a teoria de uma certa série particular de objetos: a série dos números naturais. O dilema das múltiplas reduções mostra que, no fundo, a aritmética é muito mais semelhante à álgebra do que sua apresentação superficial pode fazer parecer.

Duas décadas antes da publicação dos argumentos de Benacerraf e Parsons, os matemáticos Saunders Maclane e Samuel Eilenberg publicaram o texto "General Theory of Natural Equivalences", no qual apareceu pela primeira vez a teoria das categorias. Como é bem conhecido na literatura, há suficientes evidências de que inicialmente os criadores da teoria não a pensaram como uma 
alternativa autônoma aos fundamentos da matemática então vigentes. Pelo contrário, categorias foram introduzidas apenas como um plano de fundo axiomático para que se pudesse definir alguns conceitos, ou uma linguagem útil para a formulação de alguns problemas específicos, no campo da topologia algébrica (Marquis, 2008, p.11). Aquilo que parece ser o mais crucial turning point que haveria de realmente mudar o estatuto da teoria foi o artigo "Sur quelques points d'algèbre homologique" de Alexander Grothendieck ${ }^{3}$. Grothendieck introduziu a noção de uma 'categoria abeliana' utilizando-se de expedientes que agora são familiares a todos que já estejam habituados às teorias de topos: em vez de simplesmente caracterizar o conceito de 'grupo abeliano', ele forneceu uma lista de axiomas que descreveriam as propriedades essenciais do "universo" dos grupos abelianos conectados por seus homomorfismos (ou seja, da categoria de todos os grupos abelianos). A caracterização foi dada nos termos que chamaremos de 'puramente categóricos', isto é, tão somente em termos de 'morfismos' ou 'setas' ou com mais precisão: em termos da álgebra da operação de composição sobre os homomorfismos de um universo de estruturas. De acordo com Maclarty, a axiomatização de Grothendieck da noção de 'categoria abeliana':

[...] não é como os axiomas para grupos abelianos. Essa é uma descrição axiomática de toda a categoria dos grupos abelianos e de outros categorias similares. Não prestamos atenção ao que os objetos e setas são, apenas a quais padrões de setas existem entre objetos (McLarty, 1990, p. 356, tradução minha).

A descrição puramente categórica do universo de todos os grupos abelianos enfatizou uma suposta propriedade da teoria das categorias: sua autonomia. Essa autonomia pavimentou caminho para as diversas propostas de fundamentação da matemática feitas por William Lawvere e baseadas em teoria das categorias: teoria dos topoi, teoria elementar da categoria dos conjuntos e a teoria da categoria de todas as categorias. Pode-se sugerir que a teoria das categorias não só é autônoma no sentido de não "depender" de outras formas de se fazer matemática, mas também no sentido mais forte de fornecer ferramentas para fundamentações da matemática alternativas às teorias de conjuntos tradicionais. Essa sugestão vem de fato sendo feita por alguns autores desde a década de 60. Normalmente, ela é motivada pelo fato de que as restrições impostas pelo paradoxo de Russell tornam as teorias de conjuntos muito fracas para fornecer modelos para os casos mais interessantes de categorias. Como não parece haver maneira razoável de fornecer

\footnotetext{
${ }^{3}$ Não é meu objetivo aqui fazer uma descrição detalhada dos eventos históricos ou tampouco argumentar profundamente em defesa de afirmações sobre a história da teoria das categorias. Ao leitor interessado, recomendo artigo de Laine e Marquis: Categories in Context (2005). Os autores apresentam uma versão da história da teoria tendo como fio condutor o desenvolvimento de uma "abordagem top-down" para estruturas matemáticas.
} 
fundamentos conjuntísticos para a teoria das categorias, então alguns teóricos das categorias buscam prover fundamentos categóricos para a matemática.

Os gérmens das propostas fundacionais de Lawvere apareceram em sua tese de doutorado, Functorial Semantics of Algebraic Theories (1963), e, portanto, poucos anos antes do termo 'estruturalismo' começar a circular com mais frequência nos artigos e livros sobre filosofia da matemática. Contudo, a afinidade entre a teoria das categorias e o lema estruturalista é autoevidente. A ideia de se caracterizar os objetos em uma categoria apenas via os mapeamentos que os conectam a outros objetos dentro dessa categoria é obviamente similar a diversas formulações que encontramos para a tese estruturalista. A título de exemplo, Charles Parsons inicia desta maneira seu "The Structuralist View of Mathematical objects":

Por 'visão estruturalista' dos objetos matemáticos, quero dizer a visão de que a referência a objetos matemáticos é sempre no contexto de uma estrutura de fundo, e que os objetos envolvidos não possuem mais do que o que pode ser expresso em termos das relações básicas da estrutura (Parsons, 1990, p. 1, tradução minha).

Ao afirmar que a matemática lida com "estruturas”, uma noção de 'estrutura' deve ser fornecida, se é que a afirmação deve possuir absolutamente algum conteúdo. Assim como as teorias de conjuntos (ou, como pretendemos que fique claro ao longo do texto, até mais do que em teorias de conjuntos), a teoria das categorias é um lugar natural para se buscar uma. Há uma certa variedade de estruturalismo, batizado como 'ante rem', que se baseia na ideia de se desenvolver uma ontologia (seja axiomática ou informal) cujos objetos básicos corresponderiam explicitamente a noção de 'estrutura' e/ou a de uma 'posição abstrata em uma estrutura'. A teoria da estrutura de Shapiro (1997) e a "matemática como ciência dos padrões" de Resnik (1981) são os mais conhecidos exemplos desse tipo de abordagem. Dada a generalidade da noção de 'categoria', parece uma sugestão natural que substituamos 'estrutura' por 'categoria' e 'relação' por 'morfismo' e passemos a pensar o estruturalismo incorporado nos métodos e na linguagem da teoria das categorias como uma instância de um tal estruturalismo ante rem. Contudo, não é de modo algum claro que possamos representar literalmente qualquer tipo de modelo para uma teoria matemática como uma certa categoria. Pelo contrário, não é difícil de se imaginar alguma construção que não satisfaça a definição de categoria. Embora seja argumentável que qualquer construção possa ser definida dentro ou em termos da teoria das categorias, isso não significa, ainda, que possa ser introduzida como uma categoria tout court. $\grave{A}$ noção de 'categoria' falta a generalidade que se deve exigir de uma noção de 
'estrutura': em matemática lidamos constantemente com álgebras ou com sistemas de relações que não satisfazem a definição de categoria.

Então, se como Bell sugere, a teoria das categorias dá origem a uma "teoria axiomática geral da estrutura" (Bell, 1981, p.356), ela deve fazê-lo de uma maneira mais sútil ou "indireta". Essa maneira parece ter sido formulada mais explicitamente por Awodey (1996). Tal formulação foi motivada pelo mesmo fato que Lawvere (1966) menciona como motivação para sua teoria da categoria de todas as categorias: a crescente tendência da matemática moderna em caracterizar estruturas matemáticas em termos de mapeamentos entre objetos em vez de objetos tomados de forma isolada. Os exemplos mais paradigmáticos de categorias são sistemas de objetos matemáticos equipados com um certo tipo de estrutura, conectados por mapeamentos preservando exatamente o tipo de estrutura em questão. De fato, mesmo que haja exemplos de categorias que não se encaixem nessa descrição informal, sem dúvida essa é a ideia intuitiva que motiva a definição formal do conceito de uma categoria em geral. Se os exemplos paradigmáticos de categorias são objetos matemáticos estruturados junto com mapeamentos preservando essa estrutura, a uma certa categoria específica corresponde um certo tipo geral de estrutura. Essa certa categoria fornece uma maneira de caracterizar e descrever estrutura matemática de um certo tipo em termos de mapeamentos entre objetos matemáticos equipados com o tipo de estrutura em questão. A categoria de todos os grupos corresponde à estrutura de grupo, de todos espaços contínuos corresponde à estrutura topológica, etc. Os axiomas para a teoria geral das categorias axiomatizam precisamente a noção de um 'tipo de estrutura matemática' em termos de um "sistema de mapeamentos que preservam esse tipo de estrutura" (Awodey, 1996, p. 212).

Awodey fez em 1996 a sugestão de que filósofos e matemáticos deveriam olhar para a teoria das categorias para clarificar a noção de 'estrutura' para os propósitos de um "estruturalismo filosófico". Essa sugestão foi interpretada por alguns, talvez erroneamente, como a afirmação de que a teoria das categorias provê fundamentos para a matemática estrutural, ou pelo menos como uma consequência direta dessa asserção. Um interessante debate acerca do estatuto da teoria das categorias ocorreu entre Awodey e Geoffrey Hellman, envolvendo os textos dos autores que se encontram na referência bibliográfica do presente artigo. A tréplica de Awodey é responsável por clarificar a característica mais distintiva de uma abordagem categórica, que a diferencia dos fundamentos tradicionais da matemática. Falo da diferença entre uma abordagem top-down e bottom up para 
caracterização estruturas. Na seção seguinte a sugestão de Awodey será apresentada com mais detalhes.

\section{A SUGESTÃo DE AWODEY}

Para entendermos melhor o significado da sugestão de Awodey, talvez seja proveitoso que a coloquemos em contexto, comparando-a com outras possíveis alternativas, como a noção de 'estrutura' da teoria dos modelos. Seguindo Hale (1996) e também Dummett (1991, p.295), podemos distinguir entre dois usos para a palavra 'estrutura' já bem difundidos na literatura matemática. A teoria dos modelos nos fornece uma maneira de, dada uma certa linguagem de primeira ordem L, atribuir uma (ou frequentemente mais de uma) estrutura semântica para L capaz de tornar sentenças formuladas em L verdadeiras ou falsas. Essa estrutura é uma interpretação de L. A estrutura é introduzida como um certo conjuntodomínio não vazio de elementos, junto com relações, propriedades e operações definidas para os elementos do domínio. Todos esses diferentes itens são definidos em termos de conjuntos, e o modo como eles são "agrupados" é, por assim dizer, irrelevante. Dado uma teoria $\mathrm{T}_{\mathrm{L}}$ em L, i.e., um conjunto de sentenças formadas apenas a partir dos símbolos de $\mathrm{L}$, chamamos de um 'modelo de $\mathrm{T}_{\mathrm{L}}$ ' uma estrutura de L que satisfaça todas as sentenças de $\mathrm{T}_{\mathrm{L}}$. Então, em um certo uso de 'estrutura', dizemos que uma estrutura é uma certa coleção de elementos particulares, junto com uma lista de operações ou relações definidas entre os elementos dessa coleção. Os elementos da estrutura já possuíam seus critérios de identidade bem determinados antes de serem agrupados sob um domínio comum e, por isso, são identificáveis independentemente de sua posição na estrutura. Vamos reservar a expressão 'estrutura-modelo' para tal noção de 'estrutura'. Em outro uso bem estabelecido da palavra, que podemos chamar de 'estrutura abstrata', dizemos dessas estruturas-modelo que elas possuem uma mesma estrutura no caso de haver um mapeamento um a um entre seus domínios conservando relações ou operações relevantes em um certo contexto. Uma vez tendo fixado esse contexto, com isso fixamos critérios de identidade que nos permitem falar sobre uma estrutura abstrata particular ou em classes de estruturas abstratas.

Dummet insiste que quando o estruturalista diz que matemática é sobre estrutura, devemos interpretar o termo 'estrutura' no sentido abstrato, pois a outra opção trivializaria a tese estruturalista já que qualquer modelo de uma teoria é uma estrutura no sentido da teoria dos modelos. Hale defende que isso não é tão filosoficamente inócuo quanto Dummet pretende, pois pressupõe que uma teoria matemática é sobre seus modelos pretendidos. De qualquer modo se supormos 
que as abordagens estruturalistas resolvem o problema das múltiplas reduções, o lema estruturalista precisa ser entendido como asserindo que a matemática fala sobre estruturas no sentido abstrato.

Semelhante à noção de 'estrutura' da teoria dos modelos, há aquela que aparece nos elementos de Bourbaki. De acordo com Awodey (1996), a noção de 'estrutura' de Bourbaki é uma generalização da noção tradicional vinda da teoria de modelos, mas que aceita possíveis estruturas correspondentes a linguagens de ordem superior. De forma um pouco vacilante, Awodey chama as duas noções tanto de concepção 'modelo teorética' quanto de "noção de estrutura de Bourbaki", logo após distingui-las. Claro que isso se deve ao fato de que o aspecto relevante ao seu argumento é precisamente aquilo que ambas possuem em comum.

Awodey afirma que a noção de 'estrutura' de Bourbaki é importante por dois aspectos:

(1) A ideia de um conjunto domínio "equipado com uma estrutura" moldou a concepção standard de um objeto matemático;

(2) Ela deixou claro que ao menos alguns fatos acerca de objetos matemáticos são válidos em virtude de sua estrutura.

Evidentemente, a palavra 'estrutura' ocorre em (1) e (2) no seu sentido abstrato. Temos aqui uma tensão própria a matemática moderna. Por um lado, a concepção de estrutura de Bourbaki deixou claro que propriedades matematicamente relevantes de estruturas-modelo são precisamente aquelas que são invariantes para certos isomorfismos, ou seja, aquelas que dependem tão somente da estrutura abstrata instanciada. Apesar disso, a própria noção de 'estrutura' que foi empregada na elaboração e construção dos objetos matemáticos exige que cada objeto seja uma coleção particular construída a partir de elementos cuja identidade já era previamente determinada. "A descrição de Bourbaki de objetos matemáticos como conjuntos com estrutura leva a uma perspectiva estrutural útil, que a linguagem e os métodos da própria teoria dos modelos não servem para descrever particularmente bem." (Awodey, 2012, p. 211; tradução minha).

A tendência da matemática moderna, fortemente moldada pelos Elementos de Bourbaki, em não distinguir entre as "cópias" isomórficas de certas construções é devida ao advento do método axiomático. Há quem diga até que o dogma central do método axiomático é a ideia de que "estruturas isomórficas são matematicamente indistinguíveis em suas propriedades essenciais" (Mayberry, 1994, p.19-20). Uma teoria axiomática carrega consigo uma noção de 
'homomorfismo entre dois modelos da teoria'. Dois modelos da teoria conectados por um par de homomorfismos que são um a inversa do outro são indistinguíveis do ponto de vista da linguagem na qual a teoria em questão é formulada. Para ser um pouco mais preciso, isso significa que apenas usando as propriedades, relações e operações envolvidas na assinatura da linguagem formal dentro da qual a teoria axiomática é expressa, não podemos formular uma sentença dessa linguagem que seja verdadeira para um certo modelo $\mathrm{M}$ e falsa para um modelo $\mathrm{M}^{\prime}$ isomorfo a $\mathrm{M}$. Apesar da tendência própria ao método axiomático em identificar duas construções indistinguíveis entre si, essa ideia é literalmente falsa dentro dos fundamentos mais padrões para a matemática.

A noção categórica de estrutura seria recomendada por Awodey principalmente pelo fato de que ela se adequa melhor do que os fundamentos tradicionais a essa tendência em não se importar com propriedades que não sejam preservadas para uma certa noção de 'isomorfismo'. A teoria das categorias surgiria de uma necessidade natural por uma linguagem e por métodos capazes de expressar e lidar melhor com questões relacionadas aos diferentes tipos de estrutura. Se assim desejarmos, podemos descrever uma categoria a partir da especificação de modelos de uma certa teoria junto com os homomorfismos entre esses modelos que preservem a estrutura relevante para a teoria. Ainda assim, a categoria, tal como descrita pela linguagem puramente categórica, determina esse tipo de estrutura de uma maneira independente dos métodos iniciais de especificação (Awodey, 1996, p. 5). A descrição categórica de uma certa estrutura goza de uma "invariância sintática" no sentido de que a descrição é feita de tal modo que ela não depende de nenhuma escolha particular de alguma descrição modelo teorética possível.

Próprio ao modo modelo-teorético de se lidar com um certo tipo de estrutura é começar por uma lista de axiomas, i.e., uma teoria que caracteriza a estrutura em questão, e passar a considerar modelos que satisfazem os axiomas dessa lista, para em seguida considerar seus homomorfismos. A caracterização do tipo de estrutura em questão pode também ser feita pelo modo próprio da teoria das categorias: por meio das equações satisfeitas pelos homomorfismos da teoria axiomática da qual partimos, submetidos à operação de composição. Ou, de um modo conciso: somente pela álgebra abstrata de morfismos da categoria. A invariância sintática da teoria das categorias é creditada à linguagem que ela emprega: nenhuma propriedade que possa vir a ser definida a partir dos termos 
primitivos da teoria poderia vir a distinguir entre dois objetos isomorfos entre $\mathrm{si}^{4}$. Os argumentos que apresentaremos na seção seguinte fornecem razões para acreditarmos que mesmo que a teoria das categorias possa ser uma linguagem interessante para descrever ou iluminar aspectos de certas estruturas, ela não pode ser considerada autônoma no sentido de não depender de alguma outra teoria mais fundamental. À teoria faltaria o que se deve exigir de uma teoria fundamental da matemática.

\section{OS ARGUMENTOS DE HELLMAN CONTRA A AUTONOMIA DA TEORIA DAS CATEGORIAS}

Será importante para nossos propósitos que façamos uma distinção clara entre duas afirmações. A primeira delas, que chamaremos de tese (ou "sugestão") de Awodey, é a afirmação de que deveríamos buscar uma noção de 'estrutura' dentro da teoria das categorias, para os propósitos de uma filosofia estruturalista da matemática. Outra afirmação é a de que a teoria das categorias nos fornece instrumentais para a fundamentação da matemática de uma maneira alternativa e autônoma em relação aos fundamentos tradicionais em termos de teorias de conjuntos. Vamos chamar essa segunda afirmação de 'tese de Maclane's. Agora, podemos nos perguntar qual a conexão que existe entre a tese de Awodey e a de Maclane. De acordo com Hellman, a sugestão (ou "tese") de Awodey possuiria, naturalmente, a tese de Maclane como um pressuposto (Hellman, 2003, página 2).

Hellman começa constatando que é muito conhecido que Maclane proponha a teoria das categorias como um fundamento autônomo. Ainda assim, nas apresentações mais formais, Maclane usa a teoria dos conjuntos como fundamento. Se essa realmente for a posição oficial, então a teoria das categorias não seria de fato autônoma, por motivos óbvios. Ao mesmo tempo, contudo, Maclane sugere uma alternativa: teorias de topos podem substituir a teoria dos conjuntos em seu papel fundacional. Nesse caso, a matemática poderia vir a ser desenvolvida pela linguagem interna de um topos, constituída pelos primitivos da teoria das categorias mas "regulados" pelos axiomas adicionais em virtude dos

\footnotetext{
${ }^{4}$ Uma interessante objeção a essa afirmação foi posta por Tsementzis (2017). Segundo essa objeção a linguagem da teoria das categorias na verdade não está em posição melhor do que as teorias de conjuntos tradicionais em fornecer uma linguagem adequada ao estruturalismo, i.e., uma linguagem cujo poder expressivo seja restrito as propriedades estruturais. Note, no entanto, que a objeção se baseia em aceitar como parte da linguagem categórica nomes particulares para morfismos e objetos dentro de uma categoria. Mas é característico da teoria, desde os seus primórdios, identificar objetos e morfismos apenas via propriedades universais que só os determinam a menos de um isomorfismo. A objeção cai por terra assim que adicionamos a exigência de que não se deve empregar nomes particulares. Essa restrição não limita significativamente a utilidade da teoria, já que ela sempre é, no mínimo, um pressuposto implícito em qualquer uso da teoria.

${ }^{5}$ Hellman atribui essa tese a Maclane, no texto sobre o qual falaremos.
} 
quais uma categoria é dita ser um topos, ou, mais especificamente, um topos com o axioma de extensionalidade. Os argumentos em Hellman(2003) são apresentadas como objeções a essa alternativa, que o autor acredita que é um pressuposto da sugestão de Awodey.

A objeção que Hellman apresentou à sugestão de Awodey pode ser dividida em duas partes centrais. Primeiramente, o autor reitera o conhecido argumento de Feferman, com alguns comentários relevantes. Feferman (1977) argumenta que se espera de um fundamento da matemática que ele forneça as noções de uma coleção não estruturada e de uma operação qualquer entre duas coleções e que a teoria das categorias, assim como outras teorias algébricas, não pode fazê-lo, devendo-se antes apoiar em alguma teoria fundacional de fundo que o faça. Por fim, a objeção que parece ser a central que Hellman dirige aos fundacionalistas categóricos é denominada como 'o problema do endereço residencial'. Esse último problema gira em torno da ideia de que a teoria das categorias não poderia, por si só, fornecer bases para que se decida sobre sentenças que afirmam, de forma assertórica, a existência de estruturas tornando verdadeiras as sentenças da matemática.

É digno de nota que Hellman também faz algumas observações, ainda esquemáticas, com o objetivo de levantar dúvidas sobre o que o autor chama de uma 'autonomia forte'. A teoria das categorias satisfaria uma tal autonomia forte se a teoria não só puder fornecer fundamentos que se sustentam por si mesmos, mas também que esses fundamentos sejam capazes de um "desenvolvimento conceitual inteligível do início ao fim, genuinamente distintivo", sem qualquer desvio pela teoria de conjuntos (2003, p. 6). O ponto em se levantar essa dúvida é que não é absolutamente claro se realmente construções abstratas em termos de categorias seriam compreensíveis sem uma explicação em termos de outras teorias mais intuitivas, como as teorias dos conjuntos.

O já mencionado argumento de Feferman é apresentado em Feferman (1977) como uma objeção ao projeto fundacional de Lawvere de axiomatizar a matemática como uma teoria da categoria de todas as categorias. Ainda assim, o argumento pode ser lido de forma mais geral ${ }^{6}$. Ele se baseia na ideia de que conceitos introduzidos para se classificar estruturas, bem como o próprio conceito geral de 'estrutura', dependem definicionalmente de alguma noção prévia de

\footnotetext{
${ }^{6}$ Que a objeção tenha sido direcionada ao programa de fundamentação mencionado é explicitamente afirmado por Feferman. Meu ponto aqui é que é trivial que a objeção possa ser estendida para qualquer projeto fundacional onde as noções primitivas da teoria das categorias apareçam como as mais fundamentais da matemática - desde que aceitemos, claro, a premissa de que as noções de classe e operação devem ser propriamente explicadas dentro da teoria fundamental.
} 
operação e coleção. Dentre tais conceitos "estruturais" estariam pelo menos conceitos algébricos como grupos, anéis, etc. Mas incluiriam também o conceito de 'categoria' e, consequentemente, os conceitos mais específicos que resultam de se adicionar ao conceito de categoria determinações adicionais, como o conceito de topos.

As noções de 'coleção' e de 'operação' não só são empregadas na hora de se definir o conceito de categoria, mas também aparecem de forma crucial nas etapas posteriores de desenvolvimento da teoria, por exemplo na explicação do conceito de um funtor entre categorias. Na hora de decidir sobre questões de completude formuladas em termos de coleçôes de morfismos, muitas vezes também é necessário que se realize a operação de produto cartesiano sobre coleções de estruturas em uma categoria. Então:

[...] Em cada passo devemos fazer uso das noções não estruturadas de operação e coleção para explicar a noção estruturada a ser estudada. A prioridade, se não primazia, lógica e psicológica das noções de operação e coleção é, então, evidente (Fefernan, 1977, p. 150; tradução minha).

Feferman atribui uma espécie de prioridade psicológica aos conceitos de coleção e operação em relação aos conceitos ditos "estruturais". Pode-se objetar que tal prioridade é, em larga medida, subjetiva. Matemáticos distintos possuem intuições distintas. Matemáticos treinados de forma diferente, tendem a entender mais facilmente noções muito diferentes entre si. Mas o fato de que as pessoas primeiro aprendam um certo conceito via um outro conceito que elas já dominam, não faz com que o primeiro conceito deva necessariamente ser definido em termos do segundo. A uma tal objeção, Feferman certamente replicaria que quando ele fala em "prioridade" dos conceitos de operação e coleção em relação aos conceitos estruturais, não está falando no tipo de "prioridade cognitiva" ou "psicológica" que tenho em mente, e sim que:

[...]os conceitos gerais de operação e coleção possuem prioridade lógica com respeito às noções estruturais (tais como 'grupo', 'categoria', etc.) porque as últimas são definidas em termos da primeira e não conversamente. Ao mesmo tempo, acredito que a experiência demonstra sua prioridade psicológica. Entendo que praticantes da teoria das categorias se sentem tão em casa em seu assunto que eles acham mais natural pensar em termos categóricos em vez de termos conjunto-teoréticos, mas eu compararia isso com não precisando ouvir, uma vez que se aprendeu a compor músicas (Feferman, 1977 , p.153; tradução minha).

É plausível tentarmos responder ao argumento de Feferman sugerindo que a teoria das categorias fornece seu próprio conceito de 'operação' e 'classe' nos termos 'morfismo' e 'objeto', e esses conceitos são implicitamente definidos pelos axiomas, da mesma maneira como Hilbert sugere que os conceitos de 'ponto' e 
'reta' são definidos pelos axiomas da geometria. Um grupo de automorfismos, por exemplo, pode ser descrito, em termos conjuntos teoréticos, como um conjunto domínio $S$ equipado com um conjunto $F$ de operações unárias $S \rightarrow S$, satisfazendo os axiomas:

(i) Existe uma função de identidade em $F:\{f(x)=x \mid x \in S\} \in F$;

(ii)Toda função em $\mathrm{F}$ possui uma inversa: Se $f(x) \in \mathrm{F}$ então $f^{-1}(x) \in \mathrm{F}$;

(iii) A composição de duas funções de F também é uma função de F: Se $f(x)$ e $g(x) \in$ F então $f(g(x)) \in$ F.

Mas podemos também, equivalentemente, descrever um grupo de automorfismos como uma categoria formada por um certo objeto $S$ junto com seu morfismo identidade $\mathrm{Id}_{\mathrm{s}}$ e uma lista de endomorfismos $f: \mathrm{S} \rightarrow \mathrm{S}$ fechada sobre composição, onde todo morfismo $f$ é um isomorfismo. As "operações" mencionadas na definição conjunto teorética de um grupo de automorfismos correspondem, precisamente, a morfismos em uma certa categoria; à "classe", ou conjunto subjacente, corresponde um objeto.

É nesse ponto que Hellman faz uma contribuição à interpretação do argumento de Feferman ao notar que o problema com essa saída é precisamente a de que ela se baseia em um apelo a uma interpretação pretendida para a operação de composição, como composição de funções definidas sobre algum tipo de coleção. Hellman acredita que geralmente teóricos das categorias apresentam os axiomas como sendo puramente "algébricos". Os axiomas da teoria não são concebidos como asserindo verdades sobre algum tipo de objetos, mas apenas estipulando uma lista de condições definidoras que estabelecem os requisitos sob os quais algo pode ser chamado de uma 'categoria', assim como interpretamos axiomas de teorias algébricas. Axiomas algébricos meramente estabelecem o que significa dizer que algo é uma estrutura de um certo tipo. Se segue que os termos da teoria não necessariamente precisam ser entendidos tal como são nessa interpretação pretendida. Morfismos não precisam ser funções e, consequentemente, a 'composição' mencionada nos axiomas nem sempre se identifica com a composição entre funções em todas as possíveis interpretações da teoria.

Na verdade, o argumento de Feferman pressupõe que tratemos os axiomas da teoria das categorias como "não-assertóricos" ou algébricos, no sentido que especificamos. O ponto é que as noções de coleção e de operação seriam necessárias para que se especifique o que é que significa dizer de algo que ele satisfaz os axiomas da teoria. Classes e operações são o que constituem esse algo, 
que informalmente chamamos de 'estrutura'. Quando apresentamos os axiomas para a teoria das categorias, temos que fazer sentido da fala de que uma certa coleção equipada com uma certa operação que passa a ser designada como 'composição' forma uma categoria. O que está em jogo não seria apenas uma noção de 'classe' que possa ser fornecida de forma interna a uma categoria (pela noção de 'objeto'), mas uma que deve ser fornecida antes que se introduza a noção de 'categoria', dando sentido preciso a uma definição da forma: 'uma categoria é uma classe de objetos e uma classe de morfismos sujeitos a uma operação de composição com tais e tais propriedades...'. Uma vez que a teoria das categorias não pode ela mesma fornecer essas noções de classe e operação, seria necessária alguma outra teoria externa, que de acordo com Feferman poderia ser uma teoria extensional dos conjuntos, ou uma teoria intensional dos tipos.

O principal argumento que Hellman apresenta não se reduz a essa objeção apresentada por Feferman, embora deva estar relacionado com ela de alguma forma. A questão pode ser colocada de forma curta: “[...]De onde as categorias vêm e onde elas vivem? [...] Ou, mais formalmente, quais axiomas governam a existências das categorias ou dos topoi?" (Hellman, 2003, p. 9).

O problema aqui é o de que axiomas algébricos para a teoria das categorias, bem como axiomas para tipos específicos de categorias, não envolvem alegações existenciais assertóricas. Não se trata de dizer que nenhum quantificador existencial ocorre formalmente nesses axiomas (o que seria simplesmente falso), e nem mesmo que eles só ocorrem dentro de afirmações condicionais (o que seria verdadeiro para os axiomas universais que se aplicam a qualquer categoria, mas falso para axiomas de topos ou outros tipos de categorias que exijam a existência interna de alguns objetos universais). Se trata de uma reiteração de que mesmo as afirmações existenciais devem ser lidas, nesse contexto, ao modo algébrico e não assertórico. Para tomar um exemplo, considere o axioma da teoria de topos afirmando a existência de um objeto terminal. Tal axioma é análogo a axiomas algébricos que afirmam que estruturas de um certo tipo possuem algum elemento que satisfaça uma certa propriedade. E não é análogo a um axioma do infinito em alguma teoria de conjuntos, por exemplo, que afirma, de forma absoluta, a existência de um conjunto com uma quantidade infinita de elementos. Mas é precisamente afirmações desse último tipo que são requeridas. Axiomas algébricos podem ser usados para justificar que em uma categoria de um certo tipo existe algum objeto que satisfaz uma certa propriedade, mas não podem justificar que uma certa categoria existe, de forma absoluta. 
Uma objeção que parece quase imediata à ideia de que sejam necessários axiomas ou alguma teoria, mesmo que informal, legislando sobre a existência de estruturas matemáticas tem forte inspiração na ideia hilbertiana de verdade matemática. Uma vez que não estamos lidando com entidades concretas, parece razoável afirmar que da consistência das proposições que descrevem uma certa estrutura matemática segue-se sua existência enquanto estrutura abstrata. Hellman interpreta a afirmação de que a consistência garante a existência como um sinônimo do metateorema da completude para a lógica de primeira ordem. (Hellman, 2003, p. 11).

Duas objeções são explicitamente levantadas a essa sugestão. 1) Essa saída implica comprometer a autonomia da teoria das categorias, já que o teorema da completude deve ser provado dentro de algum framework que usualmente é a teoria dos modelos. Uma vez que a teoria de modelos seria, nas palavras de Hellman, "formalmente parte da teoria dos conjuntos" (2003, p. 11), adotar esse metateorema como a lei fundamental que governa a existência no contexto da teoria das categorias privaria a teoria de sua suposta autonomia em relação a teoria dos conjuntos. Poder-se-ia supor, então, que a solução deveria vir de uma reformulação da metalógica feita do ponto de vista categórico. Mas não se poderia apelar para os axiomas de algum topos precisamente porque esses axiomas seriam definições algébricas para um tipo específico de categoria e, portanto, qualquer afirmação derivada desses axiomas é uma asserção hipotética geral que diz que em estruturas satisfazendo certas condições algumas propriedades são satisfeitas. 2) Os modelos que são estabelecidos pelo teorema da completude não coincidem necessariamente com os modelos pretendidos. O teorema da completude para a lógica de primeira ordem não garante que a construção intuitiva que pretendemos axiomatizar "exista", mas apenas que exista alguma coisa que satisfaça os axiomas que formulamos, aceitando, por exemplo, modelos de cardinalidade superior ou inferior (portanto não isomorfos) aos pretendidos, como mostra o teorema de Lowenhein-Skolem.

Qual seria, propriamente, o problema de se assumir que toda sentença existencial da matemática é meramente uma atribuição, por assim dizer, "relativa" de existência? O problema seria o de cairmos na posição que podemos chamar de dedutivismo: toda sentença matemática, embora possa se parecer com uma verdade determinada, no fim das contas seria apenas uma afirmação hipotética que dependeria da existência das estruturas que estamos investigando. Hellman assume que uma tal posição não é aceitável pois “ameaçaria despojar a matemática 
de qualquer conteúdo distintivo" (Hellman, 2003, p.9). Uma referência é feita ao ensaio de Quine "Truth by Convention".

\section{A RESPOSTA DE AWODEY}

Mesmo que a teoria das categorias seja o framework dominante entre muitos matemáticos estruturalistas praticantes, formular sistematicamente um estruturalismo filosófico baseado em se interpretar corretamente essa prática é certamente um problema em aberto. Como temos enfatizado, a proposta de Awodey é que a teoria das categorias seja usada para fornecer uma noção precisa e flexível de 'estrutura' que se adeque melhor a prática da matemática moderna de se importar apenas com a estrutura abstrata dos objetos matemáticos. Mas essa sugestão ainda não é a mesma coisa que vindicar algum tipo de filosofia sistemática que possamos chamar de um 'estruturalismo categórico'.

Como mencionamos, o artigo de Hellman contestando a sugestão de Awodey motivou esse último a publicar outro texto como uma resposta às objeções de Hellman. Mesmo se inspecionarmos apenas o texto de 1996, onde é apresentada a sugestão de que a noção de 'estrutura' deveria ser buscada na teoria das categorias, é fácil ver que pelo menos uma parte das objeções de Hellman se baseava em alguns desentendimentos sobre o significado preciso da sugestão de Awodey. Já nesse texto, o autor tenta desvencilhar sua proposta de qualquer tipo de fundacionalismo (1996, p. 210). Também já dizia que a perspectiva estrutural endossada pela teoria das categorias poderia ser sumarizada como a afirmação de que a matemática é sobre forma invariante e não sobre um universo de objetos matemáticos que consistiriam em átomos lógicos (Awodey, 1996, p. 235).

Na resposta a Hellman, Awodey enfatiza que a proposta de se empregar a teoria das categorias para caracterizar estrutura de forma autônoma se trata de um projeto radicalmente antifundacionalista por natureza. O que está em questão não é que a teoria das categorias supostamente proveria fundamentos alternativos para a matemática, e sim uma linguagem e métodos que viabilizam a prática matemática dispensando aquilo que usualmente chamamos de 'fundamentos'.

Ao propor o problema do endereço residencial, Hellman impõe uma exigência que só faria sentido de um ponto de vista fundacionalista. Esse ponto de vista seria caracterizado pela ideia de se construir objetos matemáticos específicos dentro de um sistema fundacional particular de modo que:

(1) Haja objetos suficientes para se representar os objetos da matemática ordinária, números, espaços, álgebras, variedades, etc.; 
(2) Haja leis, axiomas e regras suficientes para se codificar todas as inferências e raciocínios que na matemática se faz sobre tais objetos.

Por outro lado, a perspectiva categórica seria caracterizada, numa primeira aproximação grosseira, por um ponto de vista que "enfatiza forma em detrimento de conteúdo, descrições sobre construções, especificação de pressuposições sobre fundamentos dedutivos, definição de propriedades essenciais em detrimento da construção de objetos satisfazendo tais propriedades" (Awodey, 2004, p. 3). Em oposição à perspectiva que satisfaz as duas exigências mencionadas acima, na maneira propriamente categórica por excelência especificamos apenas a informação ou estrutura absolutamente necessária naquele contexto, sem que se pressuponha algum "conhecimento último, especificação ou determinação completa dos objetos envolvidos" (idem). Pela linguagem e pelos métodos típicos da teoria das categorias, não haveria nenhuma necessidade de se comprometer com um universo fixo de todas as entidades matemáticas e nem mesmo com um sistema de todas as inferências.

Dizer que não há um comprometimento com um universo fixo de todas as entidades, não é o mesmo que dizer que há vários desses universos. A proposta de John Bell (1986) baseada na substituição de uma matemática absoluta interpretada sobre um universo único por uma matemática aberta a interpretação em diversos topoi correspondentes a diversas "teorias de conjuntos locais" ainda seria, essencialmente, uma proposta fundacionalista. A questão de se existem diversos universos e sistemas de inferência para a matemática seria baseada, de acordo com Awodey (2004, p.4), em uma pré-concepção fundacionalista da matemática. De forma talvez um pouco imprecisa demais, Awodey diz que a perspectiva categórica seria melhor descrita pela afirmação de que não existem tais universos e sistemas.

Essa liberação do comprometimento com algum universo fixo de entidades regidas por leis fixas torna os axiomas e teoremas da matemática "essencialmente esquemáticos". O que significaria que eles gozam de um tipo de generalidade que não resulta do emprego de quantificadores varrendo universos fixos, mas sim do fato de que nenhuma informação acerca de um universo de objetos é contida nessas afirmações, a não ser aquelas absolutamente necessárias dentro daquele contexto. Todo teorema matemático possui, mesmo que implicitamente, uma forma geral grosseiramente expressa por: "se tais e tais condições forem o caso, então tal e tal é o caso" (Awodey, 2003, p. 7).

Vamos tomar um exemplo particular qualquer de teorema da álgebra, por exemplo, o teorema de Cauchy dentro da teoria dos grupos. O teorema diz que se 
$G$ é um grupo finito e $p$ é algum número primo que divide a ordem de $G$ (ou seja, a cardinalidade do domínio de $G$ ) então $G$ contém um elemento de ordem $p$, i.e., algum elemento $x$ tal que $p$ é o menor inteiro positivo e $x^{p}$ que é igual ao elemento neutro do grupo $G$. Lembremos que a abordagem bottom up ou fundacionalista é caracterizada por começar pelo desenvolvimento de uma ontologia, cujos elementos básicos consistem em átomos lógicos a partir dos quais se constrói os objetos da matemática. Conceitos estruturais, tais como o conceito de monoide, grupo, ou mesmo categoria, são posteriormente introduzidos como uma forma de se reunir sobre um nome comum espécies de construções de acordo com sua morfologia, suas propriedades estruturais. Assim, para um fundacionalista, o teorema de Cauchy afirmaria algo da forma: "Para qualquer objeto X do domínio fixo de todos os objetos matemáticos tal que $\mathrm{X}$ seja um grupo finito vale tal e tal propriedade". A abordagem top-down reconhece que o teorema, em sua formulação geral, é mais universal do que a interpretação particular que o fundacionalista propõe dentro de seu sistema fundacional. Parafraseando Awodey para adaptá-lo ao meu exemplo: o teorema em questão não é uma quantificação universal sobre um domínio específico de objetos, pressupostos ou construídos, de alguma forma fixos e dados; o teorema em questão, embora fale sobre todos os grupos finitos, não é sobre todos os grupos dentro de um universo fixo e nem mesmo sobre todos os grupos finitos possíveis em algum domínio de todos os universos possíveis; é uma afirmação esquemática sobre uma estrutura aberta a possuir várias instâncias (Awodey, 2004, p. 5).

\section{OBSERVAÇÕES SOBRE O COMENTÁRIO DE HELLMAN À TRÉPLICA DE AWODEY}

Em um texto posterior, Hellman (2006) forneceu comentários às duas principais respostas suscitadas pelos seus argumentos contra a autonomia da teoria das categorias. "A resposta hilbertiana de Awodey", como o autor denomina a resposta que acabamos de descrever, enfrentaria um dilema: ou bem a matemática é uma mera rede formal complexa de interconexões dedutivas e conceituais, ou não. No caso positivo, de fato não precisaríamos nos preocupar com termos primitivos vindo a possuir significado dentro de axiomas assertóricos. De acordo com Hellman, nesse caso, questões de verdade que vão além da "verdade lógica" do cálculo de predicados de primeira ordem utilizado só surgiriam em contextos de aplicação da matemática onde se assumisse que as condições antecedentes em um teorema são de fato satisfeitas. Se uma tal posição ainda é 
viável depois de todas as críticas levantadas ao dedutivismo, seria uma questão em aberto.

Do outro lado da moeda, assumimos que os primitivos da teoria possuem significado, mesmo que façamos a assunção de que ele é, em algum sentido, multifacetado. A interpretação algébrica dos axiomas da teoria das categorias é, de fato, crucial para Awodey, pois é tal interpretação que confere flexibilidade à linguagem da teoria das categorias, permitindo, por exemplo, representar funções, homomorfismos, relações, etc., por meio da mesma noção de 'morfismo'. Portanto, o conteúdo semântico da noção de 'morfismo' é contextual: ele depende da categoria na qual o morfismo é incluso. Morfismos não precisam ser funções ordinárias, só precisam satisfazer os axiomas. Ao explicar o conceito de 'morfismo' dizendo que eles são qualquer coisa que satisfaça os axiomas enfrentamos um novo dilema: ou bem estamos tratando a própria noção de 'satisfação de axiomas' como primitiva, ou então novamente comprometemos a autonomia da teoria das categorias, fazendo com que a explicação do conceito de categoria dependa, no fim das contas, da noção modelo teorética de satisfação.

Quanto ao primeiro dilema, parece claro que Hellman ignorou ou não compreendeu a explicação de como a abordagem top-down não colapsa com o dedutivismo. Dedutivismo é a ideia de que toda sentença matemática é hipotética de um modo tal que sua forma aparente não deixa explícito. Para o dedutivista, todo teorema P diz na verdade que 'Se for o caso que todas as leis, axiomas e regras do sistema são verdadeiras, então P'. Consequentemente, uma sentença matemática da forma 'Se A então B' na verdade diria que 'Se for o caso que todas as leis...então Se A então B'. Críticas ao dedutivismo geralmente se baseiam na ideia de que ele torna todos os teoremas hipotéticos de tal modo que nunca poder-se-ia propriamente determinar a verdade de qualquer teorema, já que as condições antecedentes sempre permaneceriam em aberto. A leitura top-down do teorema de Cauchy que propomos alguns parágrafos acima não é hipotética no sentido dedutivista. Dissemos que o teorema é da forma 'se tal e tal ocorre, então tal e tal era o caso'. Mas as condições antecedentes não eram tomadas como afirmações assertóricas com algum valor de verdade que nunca saberemos determinar, e sim como uma lista de condições especificando o escopo de aplicação da afirmação consequente. $\mathrm{Na}$ abordagem top-down, todo teorema diz que uma estrutura de um certo tipo possui invariavelmente uma certa propriedade. Para o dedutivista, todo teorema diz que se uma lista de leis, axiomas e regras assertóricos forem verdadeiros, uma certa proposição seria verdadeira. Em suma, diferentemente do que ocorre com a interpretação dedutivista de um teorema, na interpretação topdown o teorema pode vir a possuir (e geralmente possui) um valor de verdade 
determinável, já que o papel do antecedente é apenas o de fixar sobre que tipo de construções estruturais o consequente fala.

Quanto ao segundo dilema, basta apontar que não é razoável a alegação de que estamos, no fim das contas, empregando a noção modelo teorética de 'satisfação', ou pior ainda, mobilizando uma noção indefinível. Uma das mais distintivas características da linguagem ordinária informal é a sua universalidade: ela possui uma infinidade de usos que ultrapassa o de qualquer linguagem formal. Dentre essa infinidade de usos, certamente se encontra a sua função de introduzir e explicar uma determinada teoria ou linguagem formal. Do fato de que uma certa noção informal intuitiva é empregada na linguagem ordinária ao se apresentar uma teoria formal $\mathrm{T}$ não se segue que a noção é pertinente à teoria $\mathrm{T}$ e muito menos que ela é um primitivo da teoria. Do contrário, deveríamos passar a dizer também que os conceitos de 'unidade', 'coisa', 'qualquer' são primitivos da teoria, já que dizemos 'uma categoria é qualquer coisa que satisfaça os axiomas'.

A noção de 'satisfação'na expressão 'o que quer que satisfaça os axiomas' já era empregada de forma intuitiva dentro da matemática antes de ganhar expressão formal e sistemática dentro da teoria dos modelos. E ainda hoje as noções de satisfação e modelo são usadas em contextos que a teoria formal e sistemática dos modelos não pode abarcar. Se como Hellman diz, essa teoria é formalmente parte da teoria dos conjuntos, como é que ela poderia explicar o significado de afirmações que envolvem dizer que universos de estruturas tão grandes quanto o universo de todos os conjuntos são um modelo para uma certa teoria? A matemática contemporânea possui pelo menos alguns exemplos dessas proposições 7 . Também é razoável levantar dúvidas sobre se a noção intuitiva de "satisfação" seja um primitivo formal da teoria pelo o fato de que ela é, na verdade, eliminável na expressão informal. Pode-se dizer, sem mudança de significado, 'uma categoria é...(segue-se os axiomas)', em vez de 'uma categoria é qualquer coisa que satisfaça os seguintes axiomas...'. A mesma coisa acontece com qualquer teoria algébrica.

\section{CONCLUSÃO}

A questão sobre o lugar da teoria das categorias dentro da imagem total do conhecimento humano, e não só dentro da matemática, é ainda um problema filosófico em aberto. Neste artigo lidamos com questões que dizem respeito à possibilidade de se empregar a teoria como uma linguagem autônoma para enquadrar uma concepção filosófica estruturalista dos objetos matemáticos.

\footnotetext{
${ }^{7}$ Para mencionar três exemplos: falamos sobre modelar a própria teoria das categorias; teorias de tipos; e a geometria diferencial sintética.
} 
Como uma resposta ao problema da insuficiência da teoria para decidir questões de existência no contexto matemático, reiteramos o argumento de Awodey de acordo com o qual a concepção de matemática encarnada nos métodos próprios da teoria das categorias contrasta com a concepção fundacionalista tradicional, pois nunca envolve mais do que existência contextual. Como contribuição ao debate, fizemos um esforço de mostrar que a posição de Awodey não pode ser confundida com as antigas posições dedutivistas e também que ela não pressupõe nenhum conceito de 'satisfação' primitivo ou externo à teoria das categorias, como acusado por Hellman.

\section{REFERÊNCIAS}

AWODEY, S. Structure in mathematics and logic: A categorical perspective. Philosophia Mathematica, v. 4, n. 3, 1996. pp. 209-237.

An Answer to Hellman's Question: Does Category Theory Provide a Framework for Mathematical Structuralism?. Philosophia Mathematica, v. 12, n. 1, 2004. pp. 54-64.

BELL, J. Category Theory and the Foundations of Mathematics. The British Journal for the Philosophy of Science, Vol. 32, No. 4.,1981, pp. 349-358.

. From absolute to local mathematics. Synthese, v. 69, 1986, pp. 409-426.

BENACERRAF, P. What numbers could not be. Philosophical Review, vol. 74, n. 1, 1965, pp. 47-73.

CAMPBELL, H.E. The structure of arithmetic. New York: Appleton-Century-Crofts, 1970.

CARTER, J. Structuralism as a philosophy of mathematical practice. Synthese, v. 163, n. 2, 2008, pp. 119-131.

CHANG, C., KEISLER, H. Model Theory. 3rd enl. ed. New York: Elsevier, 1990.

DUMMETT, M. Frege: Philosophy of mathematics. Melksham: Redwood Press Ltda. 1991.

EILENBERG, S.; SAUNDERS, M.L. General Theory of Natural Equivalences. Transactions of the American Mathematical Society, Vol. 58, No. 2, 1945, pp. 231-294.

FEFERMAN, S. Categorical foundations and foundations of category theory. In: Logic, foundations of mathematics, and computability theory. Dordrecht: Springer, 1977. pp. 149-169.

GROTHENDIECK, A. Sur quelques points d'algèbre homologique. Tohoku Mathematical Journal, Second Series, v. 9, n. 2, 1957, pp. 119-183.

HALE, B. Structuralism's unpaid epistemological debts. Philosophia Mathematica, v. 4, n. 2, 1996.p. 124-147.

HELLMAN, G. Does category theory provide a framework for mathematical structuralism?. Philosophia Mathematica, v. 11, n. 2, 2003, pp. 129-157. 
What is categorical structuralism?. In: van Benthem, J. et al (eds.). The Age of Alternative Logics. Dordrecht: Springer, 2006. pp. 151-161.

LANDRY, E., MARQUIS, Jean-Pierre. Categories in Context: Historical, Foundational, and Philosophical. Philosophia Mathematica, v.13, n.1, 2005, pp.1-43

LAWVERE, F. W. Functorial semantics of algebraic theories. Proceedings of the National Academy of Sciences of the United States of America, v. 50, n. 5, 1963, p. 869.

. The category of categories as a foundation for mathematics. Proceedings of the conference on categorical algebra, 1966, p. 1-20.

An elementary theory of the category of sets (long version) with commentary. Reprints in Theory and Applications of Categories, v. 11, 2005, p. 1-35.

MARQUIS, J.-P. From a geometrical point of view: a study of the history and philosophy of category theory. New York: Springer Science \& Business Media, 2008.

MAYBERRY, J. What is Required of a Foundation for Mathematics?. Philosophia Mathematica, v. 2, n. 1, 1994, pp. 16-35.

MCLARTY, Colin. Exploring categorical structuralism. Philosophia Mathematica, v. 12, n. 1, 2004. pp. 37-53.

The uses and abuses of the history of topos theory. British Journal for the Philosophy of Science, v. 41, n.3, 1990. pp.351-375.

PARSONS, C. Frege's Theory of Number. In: Black, M.; Alston, W.P. (eds.). Philosophy in America. Ithaca: Cornell University Press, 1964, pp. 180-203.

RESNIK, M. Mathematics as a Science of Patterns: Ontology and Reference. Noûs, Vol. 15, No. 4, Special Issue on Philosophy of Mathematics, 1981, pp. 529- 550.

SHAPIRO, S. Philosophy of Mathematics: Structure and Ontology. New York: Oxford University Press. 1997.

TSEMENTZIS, D. Univalent foundations as structuralist foundations. Synthese, v. 194, n. 9, 2017, p. 3583-3617. 